\title{
Fluorescence Rise time Measurements for High Temperature Fluorescence-Based Thermometry
}

\author{
S.W. Allison ${ }^{*}$, S.M. Goedeke ${ }^{*}$, M. R. Cates, W.A. Hollerman ${ }^{* *}$, \\ J. I. Eldridge*** and T. J. Bencic ${ }^{* * *}$ \\ * Engineering Science and Technology Division, Oak Ridge Nation Laboratory, \\ Oak Ridge, TN 37831 \\ ** Department of Physics, University of Louisiana at Lafayette, Lafayette, LA 70504 \\ *** Optical Instrumentation Technology Branch, NASA Glenn Research Center, \\ Cleveland, $\mathrm{OH}$.
}

\begin{abstract}
Certain ceramic-like phosphor materials exhibit bright fluorescence with a pronounced temperature dependence over a range which spans the cryogenic to $1700 \mathrm{C}$, depending on the specific phosphor. To measure temperature, a surface, for instance a turbine blade, is coated with the material. An optical system, sometimes including optical fibers, conveys stimulating light and collects the emission for analysis. Either emission intensity or decay time may indicate temperature. Previously fielded tests have involved surfaces such as blades, vanes, pistons, in-take valves, sheets of galvanneal steel, etc. The fluorescent coatings may be applied to small parts via sputtering methods or to large areas by mixture with inorganic binders. Presented here are results characterizing fluorescence rise times as a means of determining temperature from ambient to $700 \mathrm{C}$ for $\mathrm{Y}_{2} \mathrm{O}_{3}$ : Eu.
\end{abstract}

\section{BACKGROUND}

Phosphor materials are fine-grain particles that efficiently luminesce when stimulated by a suitable energy source. An excitation source may be electromagnetic (X-ray, ultraviolet, visible, or infrared) or it may be particle radiation (electron or proton beam). Some phosphor materials are ceramics and are thus very durable and impervious to degradation in high temperature environments. These materials can be used as temperature indicators by coating them onto a surface of interest, stimulating the characteristic fluorescence and analyzing the characteristics of the emitted light (1) Several fluorescence attributes may change with temperature: emission intensity, spectral distribution, line width, line position, decay time, and rise time. For circumstances involving imaging, intensity is often used as the temperature indicator. For point measurements, the decay time is often the best choice as it is usually the most sensitive parameter to temperature. In the present work we describe an 
investigation concerning the use of rise time as a temperature indicator.

The most usual time dependence for short pulsed excitation is given by:

$$
I=I_{0} \exp (-t / \tau)
$$

where $I_{0}$ is the initial amplitude and $\tau$ is termed the decay time. It is a sensitive function of temperature over a given range. Figure 1 illustrates a general setup for effective temperature measurement with surface coated with phosphor. The illustration shows a laser, but there has been increasing use of light emitting diodes (LEDs) for some situations.

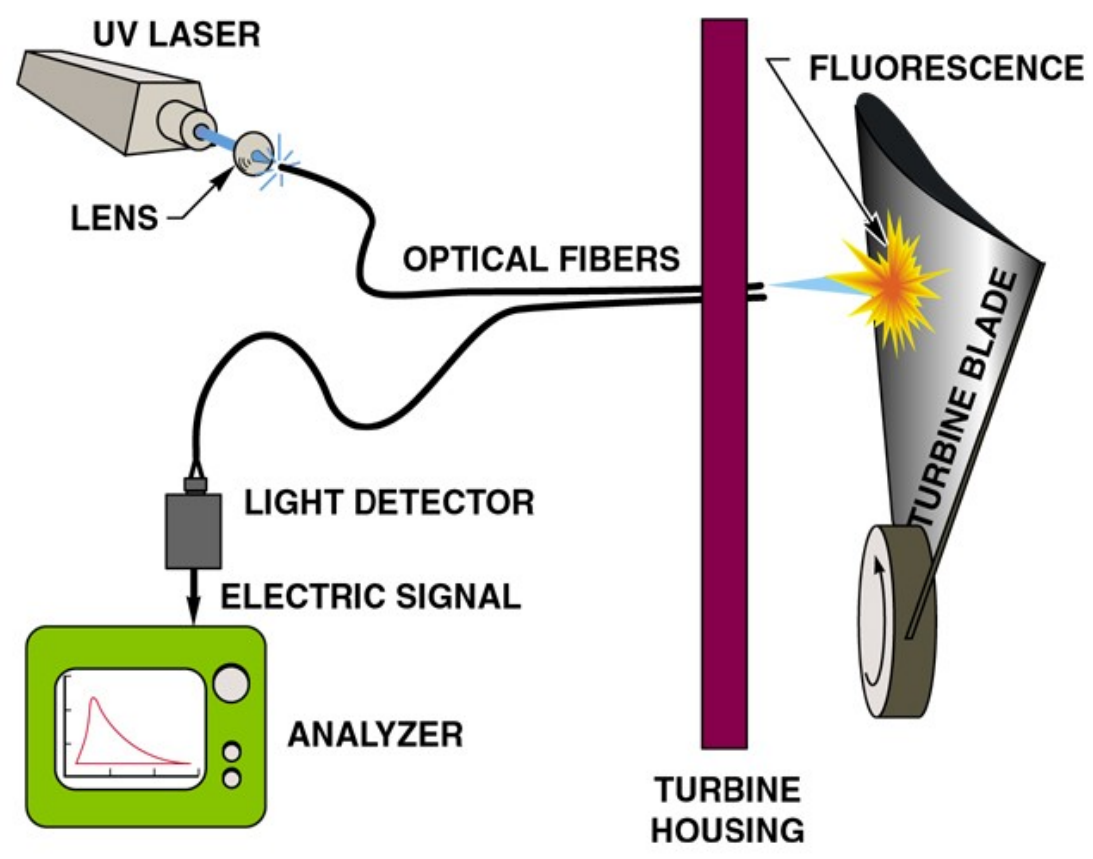

Figure 1. Representative thermometry system showing light source, light delivery and collection, a coated moving target, detection and data anaysis system.

One of the most important materials for high temperature phosphor thermometry is $\mathrm{Y}_{2} \mathrm{O}_{3}: \mathrm{Eu}$. Fluorescence from some coupons coated with a mixture of this material and an inorganic binder are shown in Figure 2. These samples were exposed to 1 hour at 1200 and $1300 \mathrm{C}$ respectively in order to assess the coating durability and high temperature capability. The red fluorescence is stimulated by a ultraviolet lamp. Sputtering methods can sometimes be used to achieve a durable phosphor coating. However, there are some situations where sputtering is impractical such as when the test area is rather large or when there is not enough time to schedule people and sputtering equipment. Several binder materials appear to work up to $1200 \mathrm{C}$ and we have had some success to as high as 1500 C. References 2-4 describe binder studies aimed at these high temperatures. 


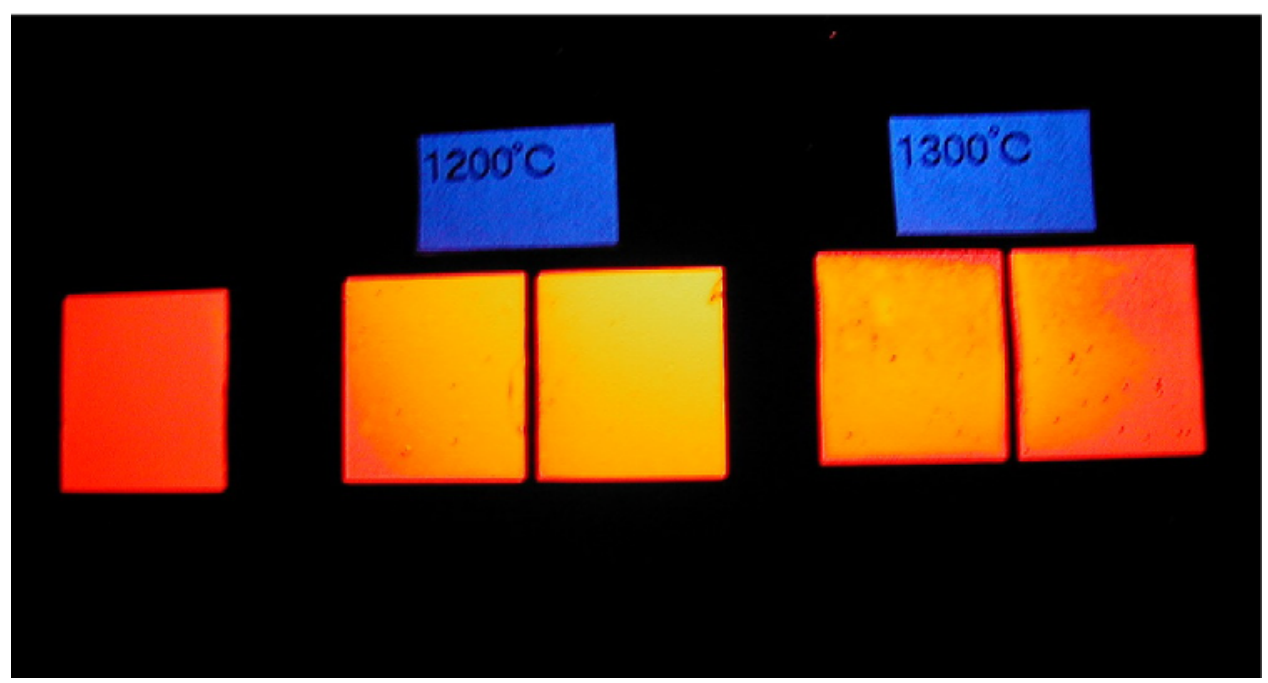

Figure 2. Fluorescence from ceramic coupons coated with $\mathrm{Y}_{2} \mathrm{O}_{3}$ :Eu phosphor.

Excited by UV lamp. Samples treated 1 hour 1200 and 1300 C, respectively.

\section{Rise Time}

The decay time of $\mathrm{Y}_{2} \mathrm{O}_{3}: \mathrm{Eu}$ is a sensitive function of temperature above about 600 $\mathrm{C}$ and it has been used effectively for thermometry above that value. RhysWilliams et al (5) noted that this emission also has a rise time associated with it that depends on Eu concentration. Their rise time determinations ranged from about 60 microseconds at room temperature for a 5.56 mole \% sample to 320 microseconds for a 0.27 mole \% sample. Ranson et al (6) indicated that the risetime from this phosphor material may be used for temperature sensing. They derived a model for the emission time dependence and give data for a particular dopant concentration, 3.2 mole $\%$. They communicate that the rise time is temperature dependent from at least ambient temperatures to $800 \mathrm{C}$. Thus it enables this already useful material to cover a much wider temperature range. The dominant emission arises from direct excitation, denoted by $n_{1}$ in the equation below and by feeding from a higher excited state $n_{2}$. The instantaneous population from the emitting state is according to Ranson:

$n_{\text {total }}=\left[n_{1}+n_{2}\left(1-\exp \left\{\frac{-t}{\tau_{\text {Rise }}}\right\}\right)\right] \exp \left\{\frac{-t}{\tau_{\text {Decay }}}\right\}$

We have made a detailed study of the temperature dependence from a crystalline sample of $\mathrm{Y}_{2} \mathrm{O}_{3}$ :Eu with the dopant at $0.5 \%$. 


\section{EXPERIMENT}

For the measurement of emission versus temperature, a nitrogen laser of 3 ns duration at $337 \mathrm{~nm}$ excited the sample. The sample was about $5 \mathrm{~mm}$ across. At this wavelength, the excitation efficiency at room temperature is pretty low, a crystalline sample emits more fluorescence than a powder sample. A 1 mm diameter sapphire rod collects the fluorescence and directs it to a photomultiplier. The signal is viewed on a digital oscilloscope which communicates the digitized trace to a laptop. Figure 3 shows two example traces at 22 and $300 \mathrm{C}$ respectively. The initial spike in the signal is due to laser light leaking through the fluorescence band pass filter. It is commonly the case that this feature is difficult to remove completely for practical experiments. So, it is removed from the data and ignored in the analysis. The characteristic rise and fall of the fluorescence are clearly illustrated here. The overall increase in signal as temperature is increased is due to the fact that the absorption cross section for the nitrogen laser excitation wavelength increases with temperature.

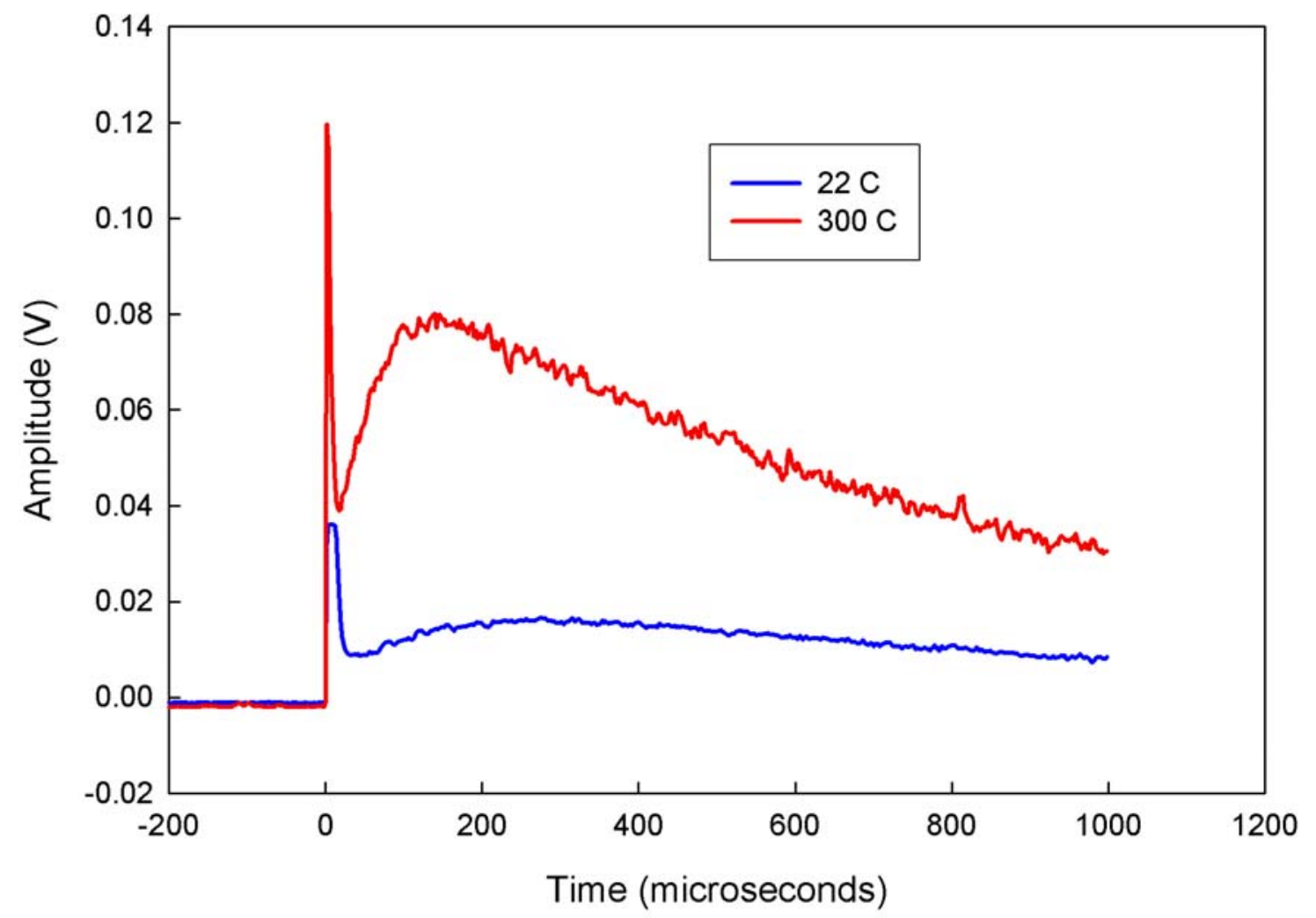

Figure 3. Emission amplitude versus time for $\mathrm{Y}_{2} \mathrm{O}_{3}: \mathrm{Eu} 0.5 \%$.

Figure 4 shows the emission for temperatures ranging from 22 to $300 \mathrm{C}$.
The initial laser spike is removed. It is seen that the rise time gets shorter as 
temperature is increased. Each trace has been fit to the Ranson equation using a regression fitting routine in Sigma Plot 8.0.

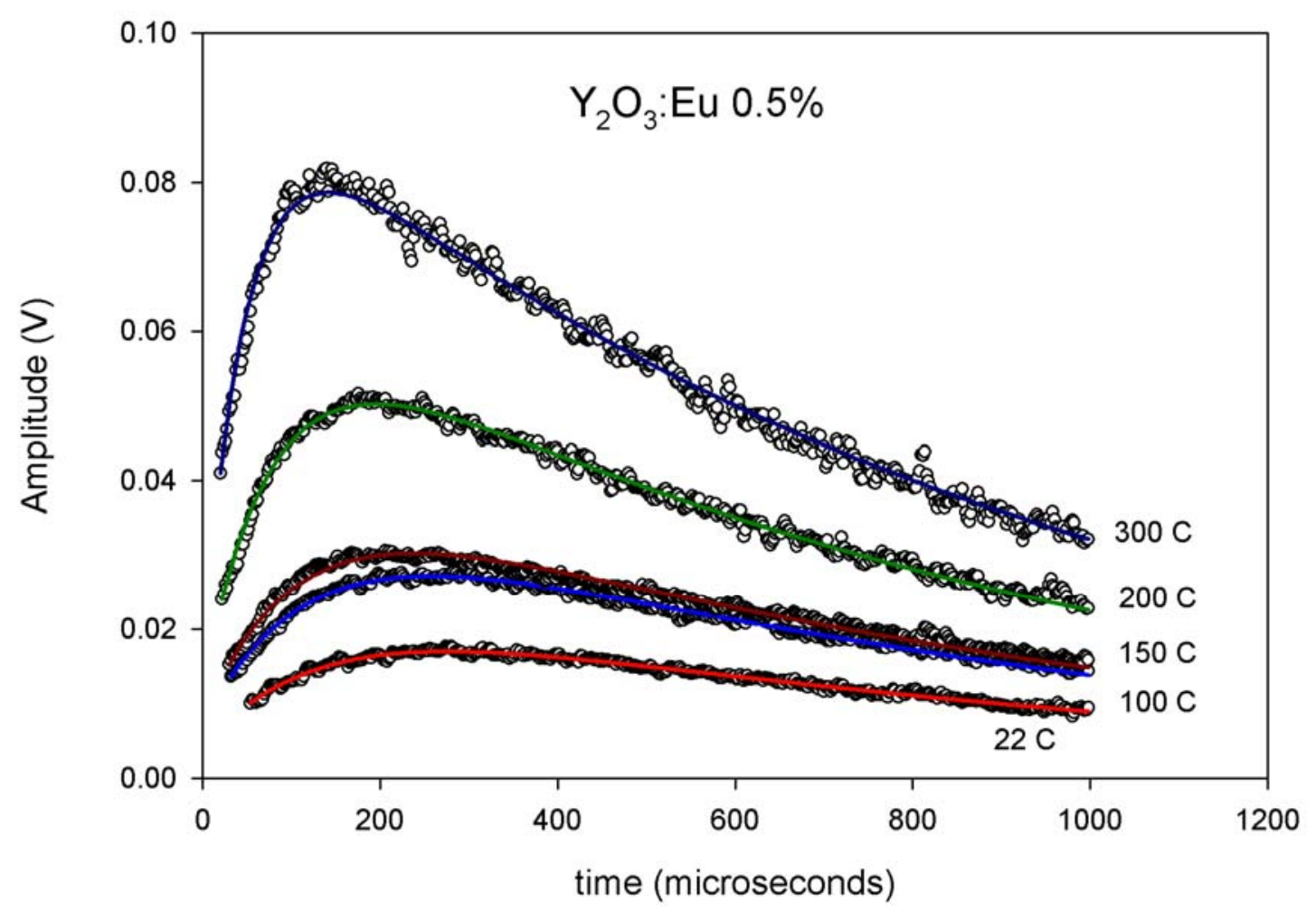

Figure 4. Emission Amplitude versus time for 22 to $300 \mathrm{C}$.

To display the dependence at higher temperatures, the time base was expanded and the results are plotted in Figure 5. From previous experience, the decay time is approximately constant to about 550 to 600 C. Thus, only the 700 $\mathrm{C}$ data shows a decrease in decay time.

The rise time obtained from the fit is plotted in Figure 6 versus temperature. The rise time changes by an order of magnitude over the entire region of temperature. This is not as great a temperature dependence as decay exhibits in some instances. It is nonetheless a useful degree of temperature dependence. It extends the range over which this already useful material may be used.

Given the fact that the room temperature rise time increases as dopant concentration decreases, it may be that a lower dopant concentration exhibits an increased temperature sensitivity. This may be explored in future work as we have samples as low as $0.1 \%$ dopant concentration. The drawback is that signal levels are low. 


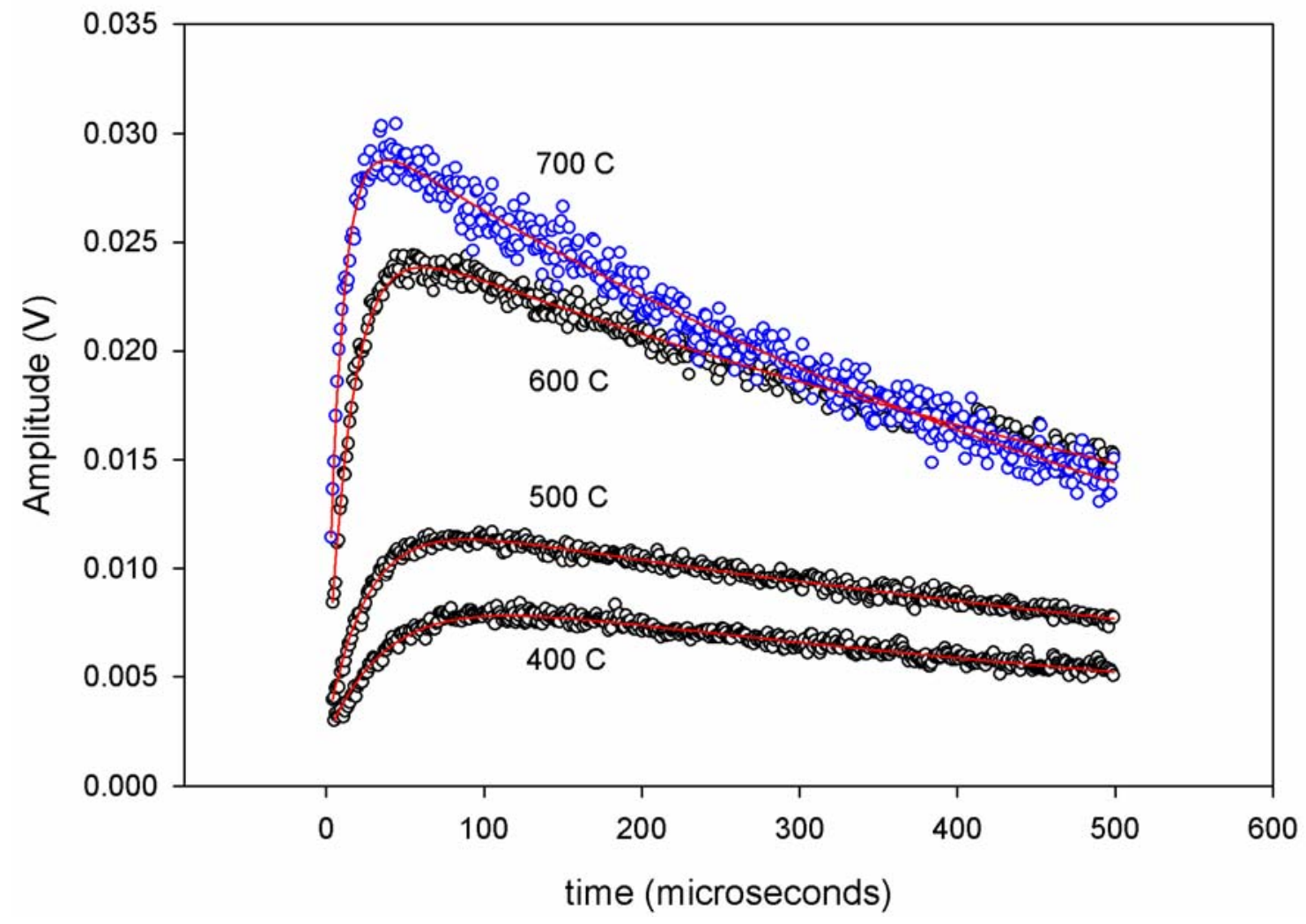

Figure 5. Emission amplitude versus time for 400 to $700 \mathrm{C}$.

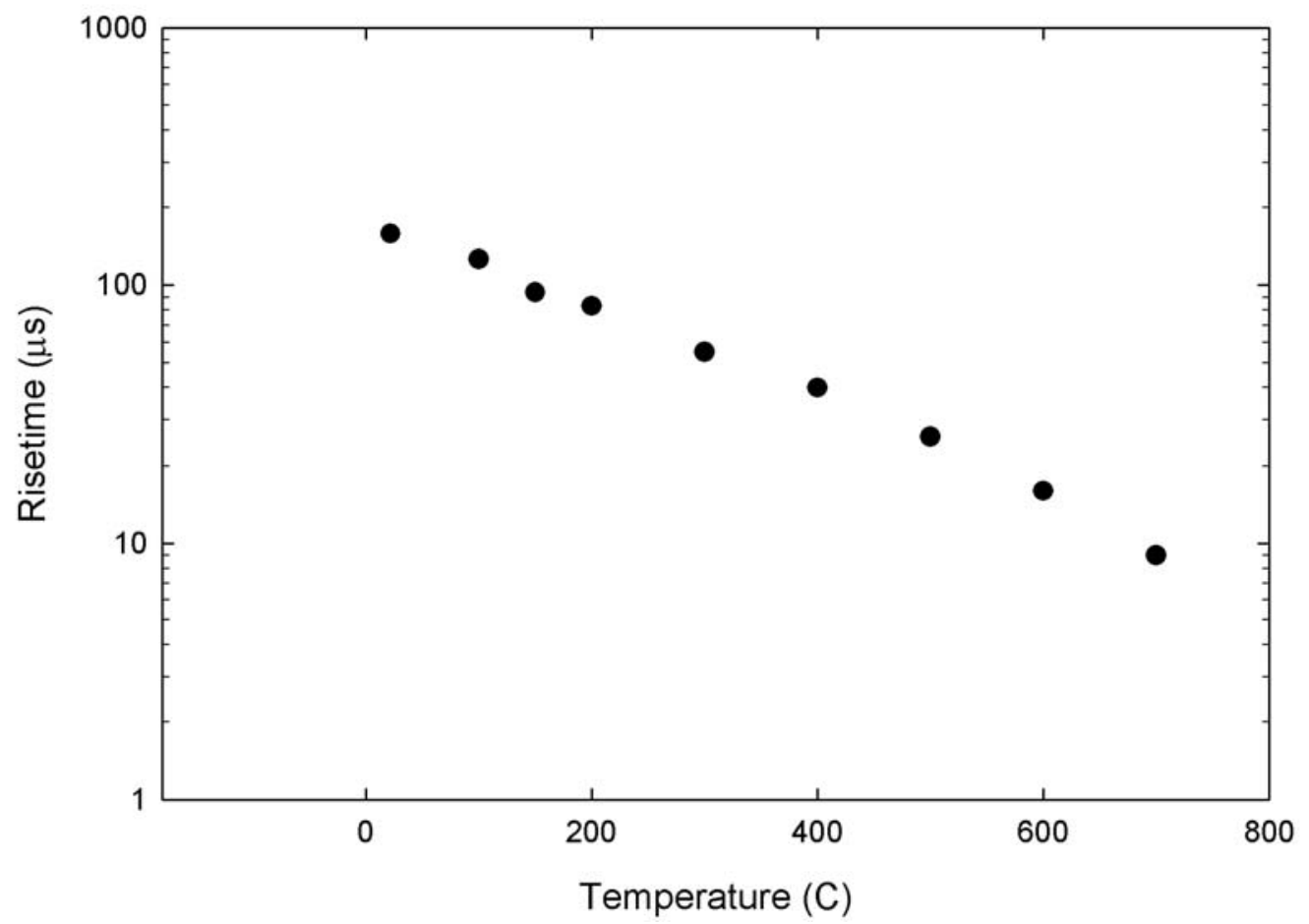

Figure 6. Risetime versus Temperature. 
It may also be instructive to examine other materials. YAG:Eu is a material that has shown temperature dependence to approaching $1400 \mathrm{C}$. Its decay time only begins to change at around $700 \mathrm{C}$. If its rise time shows a similar temperature dependence, its useful range may also be similarly extended.

\section{CONCLUSIONS}

The record temperature in our lab for observing fluorescence is1700 C. This was achieved with a YAG:Dy phosphor. Though this material apparently does not display a useful rise time, it could be mixed with $\mathrm{Y}_{2} \mathrm{O}_{3}: \mathrm{Eu}$ and the result would be a fluorescent coating which could cover an exceedingly wide temperature range. The rise and decay times of the Eu material could be monitored at $611 \mathrm{~nm}$ up to about $1200 \mathrm{C}$. The Dy fluorescence at $453 \mathrm{~nm}$ could be monitored for its temperature dependence from about $800 \mathrm{C}$ and above. The results here clearly point to the utility of using rise time for temperature measurement. It is yet another resource in a growing toolkit for fluorescence-based thermometry.

1. S.W. Allison and G.T. Gillies, "Remote Thermometry with Thermographic Phosphors Instrumentation and Applications", Rev. Sci. Instrum, 68 (7), 2615-2650, 1997.

2. S.W. Allison, D.L. Beshears, T. Gadfort, T. Bencic, J. Eldridge, W.A. Hollerman, and P. Boudreaux, High Temperature Surface Measurements Using Lifetime Imaging of Thermographic Phosphors: Bonding Tests, 19th International Congress on Instrumentation in Aerospace Simulation Facilities, August 27-30, 2001.

3. W. A. Hollerman, S. W. Allison, D. L. Beshears, R. F. Guidry, T. J. Bencic, C. R. Mercer, J. I. Eldridge, M. R. Cates, P. Boudreaux, and S. M. Goedeke, "Development of Fluorescent Coatings for High Temperature Aerospace Applications," Proceedings of the 2002 Core Technologies for Space Systems Conference Proceedings," Colorado Springs, CO., November 19-21,2002.

4. J. I. Eldridge, T. J. Bencic, S. W. Allison, and D. L. Beshears, Depth-Penetrating Temperature Measurements of Thermal Barrier Coatings Incorporating Thermographic Phosphors, Journal of Thermal Spray Technology, 13(1), pp. 44-50(7), 1 March 2004.

5. A. T. Rhys-Williams and M. J. Fuller, "The use of a Computer Controlled Luminescence Spectrometer in the Study of the Time Resolved Emission of Trivalent Europium in Yttrium Oxide," Computer Enhanced Spectroscopy 1, 145 (1983).

6. R. M. Ranson, E. Evangelou, and C. B. Thomas, "Modeling the fluorescent lifetime of $\mathrm{Y}_{2} \mathrm{O}_{3}: E u$ " Applied Physics Letters, Vol 72(21), pp. 2663-4, 1998.

\section{ACKNOWLEDGEMENTS}

Oak Ridge National Laboratory is operated for the U.S. Department of Energy by Oak Ridge National Laboratory, managed by UT-Battelle, LLC, for the U.S. Department of Energy under contract DE-AC05-00OR22725. The authors are also grateful to NASA Glen Research Center which funded a portion of this effort. 